\title{
IUMRS-ICA-98 Meeting in Bangalore Explores Wide Range of Materials: Soft to Superhard, Semiconducting to Superconducting, and Simulated Materials
}

The 5th IUMRS International Conference in Asia (ICA) was held in Bangalore, India, October 13-16, 1998 in association with the International Union of Materials Research Societies (IUMRS), the Indian Institute of Science (IISc), and Jawaharlal Nehru Centre for Advanced Scientific Research (JNC). Hosted this year by MRS-India (MRS-I) and IISc, this conference continues the series of the International Conferences in Asia sponsored by IUMRS. These conferences are held every other year in Asia to foster communication of ideas and achievements in materials science and engineering in Asia. Over 700 delegates attended ICA98 , mostly from India but with about 100 delegates from 14 other countries. The conference had nine plenary talks and 22 theme symposia in seven parallel sessions and three afternoon poster sessions. A workshop and panel discussion on materials science education in India complemented the meeting. Lunches of Indian cuisine, coffee breaks, an elaborate evening banquet, and a cultural performance were provided for all delegates, representative of the warm hospitality that greeted all attendees. The conference concluded with a presentation of the Young Research Awards (see sidebar), with the room still packed with attendees on the last day.

C.N.R. Rao, President of JNC and founding president of MRS-I, opened the inaugural session. He stressed the role of advanced materials in fundamental research and technology, stating that materials research fuels other rapidly advancing fields such as information technology and biotechnology. He described how technology affects every person, noting that even the poorest towns of India have transistor radios. P. Rama Rao (Chair of the IUMRSICA-98 Program Committee) gave a brief account of the proposed program. R. Chidambaram, President of MRS-I, highlighted the activities and objectives of MRS-I. M. Doyama, Immediate Past President of IUMRS, stressed the necessity of international links in the area of materials science and delineated the efforts of IUMRS in this regard. G. Mehta, Director of IISc, welcomed the delegates to the Institute and expressed hope that the Conference would bring together scientists and technologists. S.V. Subramanyam, General Secretary of MRS-I, proposed a vote of thanks to all.

\section{Plenary Presentations}

Three plenary sessions, with several talks each, covered a diversity of topics

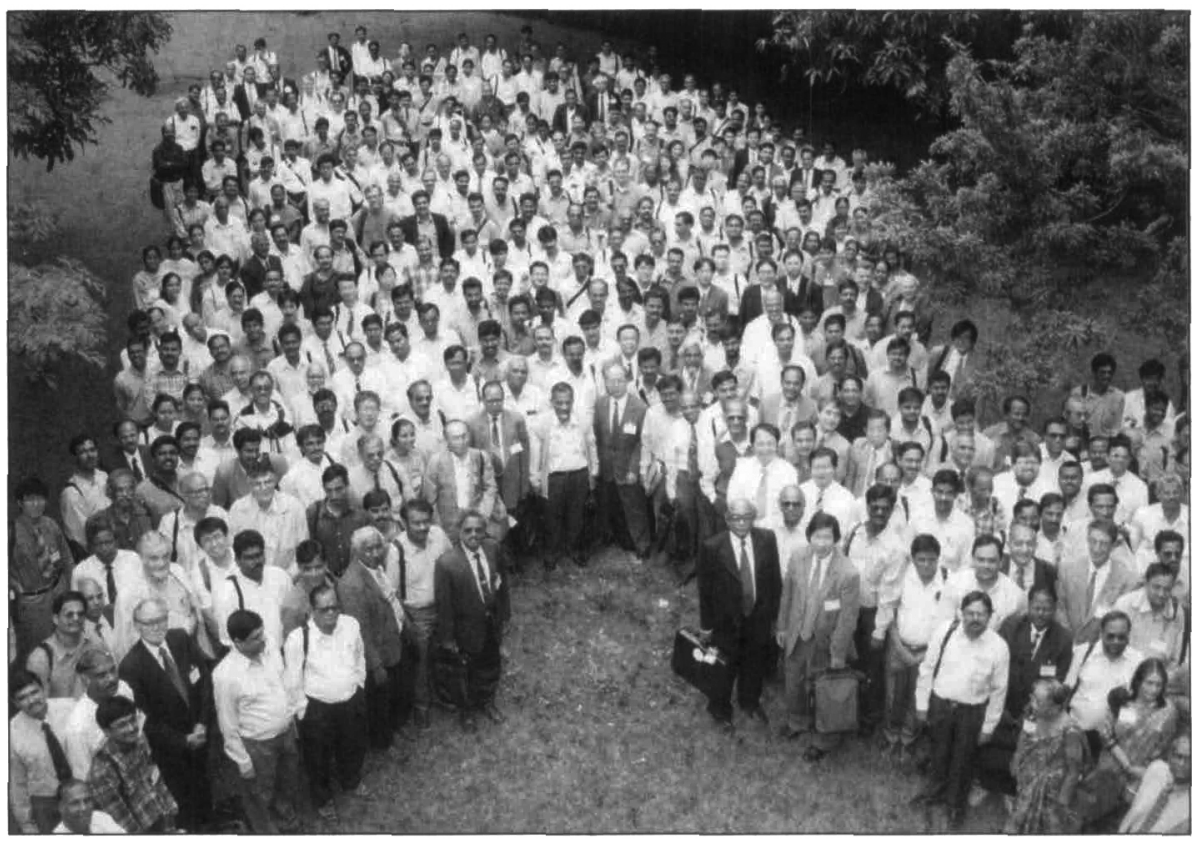

Over 700 delegates attended the 5th International Union of Materials Research Societies International Conference in Asia (IUMRS-ICA), held in Bangalore, India, October 13-16, 1998.

and provided a flavor of the conference. In the first plenary session, C.N.R. Rao (JNC), talked about "Some New Directions in Chemistry," covering a range of topics from synthesis and structure to dynamics and properties, but emphasizing new and metastable materials using soft chemistry. New chemistry focuses on the use of weak bonds such as van der Waals bonds, hydrogen bonds, and electrostatic interactions. Another important area Rao covered was porous solids, describing the many shapes and size of pores that can be constructed and how these pores can control flow through the materials.

M. Parrinello (Max-Planck-Institut), a world leader in computer simulation and molecular dynamics, covered "PressureInduced Disorder and Frustration in Hydrogen-Bonded Crystal." By varying the external pressure, researchers can explore and understand the action of the hydrogen bond. He used ice as the test material, and complemented $a b$ initio molecular dynamics simulations with experiments. Using the recently developed $a b$ initio path-integral method, Parrinello was able to assess the relevance of quantum effects such as tunneling and zero-point vibrations.

Taking pressure to higher orders, R. Chidambaram (Chair, Atomic Energy
Commission, India) covered, "Materials Response to High Pressures." In addition to basic pressure studies, he described the importance of knowing exactly how materials will react under explosive conditions, such as for nuclear technology. A combination of experimental techniques, firstprinciple theories, phase transformation studies, interpretation of shock temperatures, and the calculation of the equation of state can help augment this basic understanding

Looking at the science and the art of tailoring or designing solid materials, J. Etorneau (Institut de Chimie de la Matiere Condensee de Bordeaux) spoke on "Novel Synthesis Methods for New Materials in Solid State Chemistry," focusing on methods of synthesis that can happen at low temperatures. Chemical reactions are like a black box where reactants go in and products come out, and the task is to figure out what happens in the middle, whether stable or metastable materials form, or maybe nothing happens at all. Etorneau covered intercalation of oxygen atoms by electrochemical and other methods, and also novel methods such as the use of supercritical fluids as solvents for preparing nitrides and fluorides. Noting that most materials are discovered by accident, he mentioned the emerging 


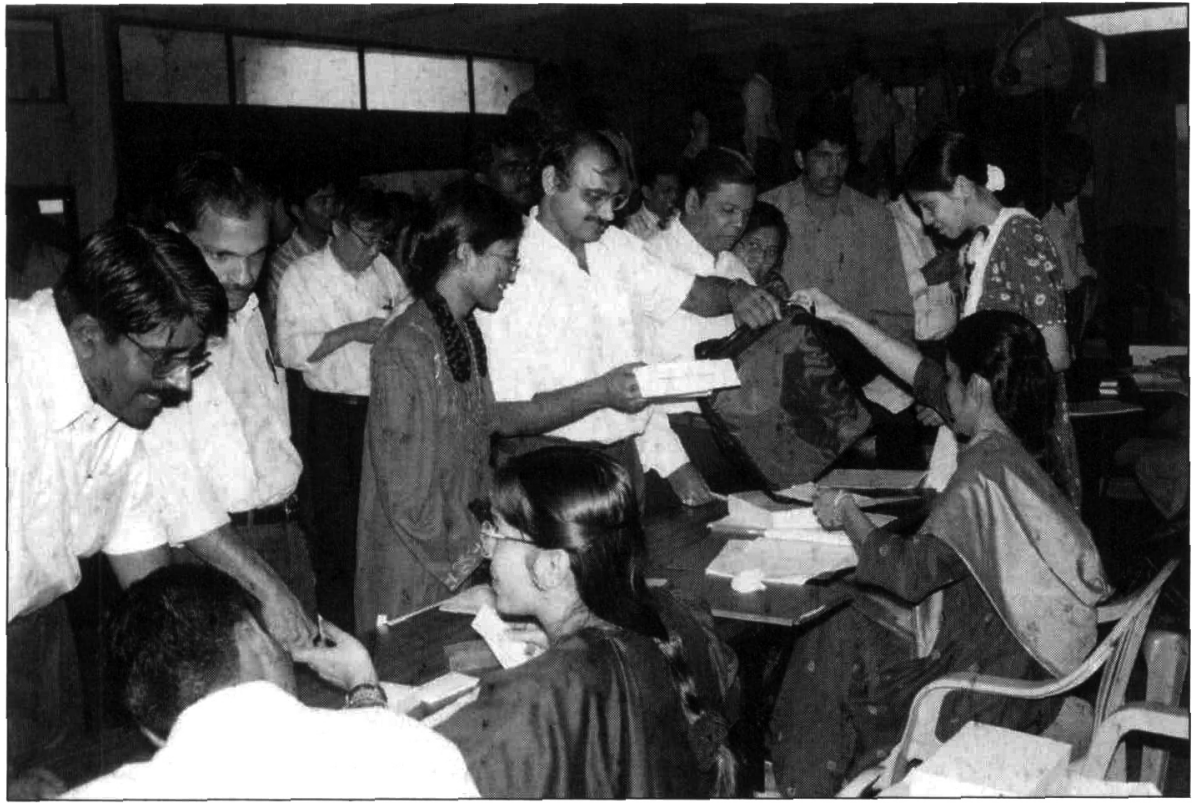

Attendees register at the 5 th International Union of Materials Research Societies International Conference in Asia (IUMRS-ICA), which convened on October 13, 1998 in Bangalore, India.

importance of combinatorial chemistry that may herald a revolution in searching for new molecules.

Expanding on the theme of low-temperature synthesis, J. Livage (Universite Pierre et Marie) presented "Sol-Gel Synthesis of Advanced Materials." Using "chimie douce" (soft chemistry), shaped materials can be made directly from solution, coatings and membranes can be synthesized, and organic-inorganic hybrids can be combined in the same beaker. In the optical arena, organic dyes can be encapsulated in silica. Also cells or other biological matter can be entrapped within a sol-gel matrix to be used for molecular recognition or to detect disease.

In a presentation on "Materials for R\&D Space Applications," K. Kasturirangan (Director, Indian Space Research Organization [ISRO]) described strides made by the launch vehicle and satellite programs of ISRO, covering telecommunications, structural elements of the launch vehicle, thermal elements, propulsion materials, and other special materials. Current efforts are directed toward development of alloy systems with improved specific strength and specific stiffness, increased service temperature of titanium alloys by moving toward titanium-based intermetallics, overcoming the brittleness of ceramics through development of ceramic-matrix composites, and development of special coating processes for dedicated applications.

Robert W. Cahn (Cambridge University), in his talk on "Disordering and Reordering of Mechanically Milled Superlattice Alloys," described mechanically disordered intermetallic systems formed via milling. Using this method, states of order can be achieved that cannot be obtained by heat treatment. He described how microhardness increases to a peak as the material is milled (and as it becomes more disordered), and then it drops. The lattice parameter generally increases with increased disorder and keeps changing well after long-range order is gone, due to continuing loss of short-range order. Also, the degree of lattice parameter change is related to the difference in the size of the component elements, but not due to their absolute sizes.

D. Shechtman (Technion) talked about the interesting and unexpected history of quasicrytals. When Shechtman first proposed that the 10-fold diffraction pattern he observed was a real crystal and not just an artifact of twinning, others thought it was nonsense. Now quasicrystals are found to be common and easy to make. The discovery of quasicrystals ended the equality between periodicity and order. Quasicrystals have order, but are not periodic as all previously known crystals were (by definition). Now, Shechtman said, the definition of a crystal is more a function of how discrete the diffraction pattern is. In a later presentation, Shechtman described how quasicrystals can be put down by plasma spray methods to produce lowfriction surfaces. Large quantities of many types of samples can now be made. The next step is choosing a single composition and optimizing coatings by varying process conditions.

H. Mori (Osaka University) delivered a plenary talk on "In-Situ TEM Observation of Spontaneous Alloying in NanometerSized Particles" The features of spontaneous alloying were discussed and the following results were summarized: Spontaneous alloying becomes more difficult to take place with increasing particle size. Spontaneous alloying takes place via a solid-state process. Electron illumination is not prerequisite for spontaneous alloying. Spontaneous alloying is not an artifact originating from the temperature rise in $\mathrm{nm}$ sized particles which might be induced by heat of condensation. The critical size of initial particles below which alloy particles are successfully formed by spontaneous alloying increases with increasing heat of alloy formation. Mori suggested from these results that in nm-sized ultrafine particles chemically driven atom migration may take place to some extent.

\section{Technical Sessions}

The conference covered a broad spectrum of experimental and theoretical developments on materials with the hope of enhancing researchers' fundamental understanding of materials and helping them to identify material processes for technological applications. Topics ranged from structural materials and coatings to electronic, optical, and biomaterials.

\section{Structural Materials and Coatings}

Composite materials (covered in Symposium T) have become important in aerospace, pressure vessel, and civil engineering applications. Fiber-reinforced polymer composite materials are now widely used, while the future holds hope for metal-matrix and ceramic-matrix composites. There is much effort in India to produce carbon fiber, and in the next century India is expected to have indigenous fibers. A significant aspect of the session was bringing together materials scientists concerned with development of fibers, matrix materials, toughening agents, and mechanical property evaluation of composites. The session provided a forum for deliberations about availability of materials, addressing cases where lack of availability is considered a major block to future developments.

Symposium $O$ on Intermetallics started with an assessment of aluminides for structural applications. Also covered was the effect of alloying on hydrogen embrittlement and other processing/properties correlations. Another group of talks focused on TiAl and the effect of stress relaxation, dislocations, and phase transi- 


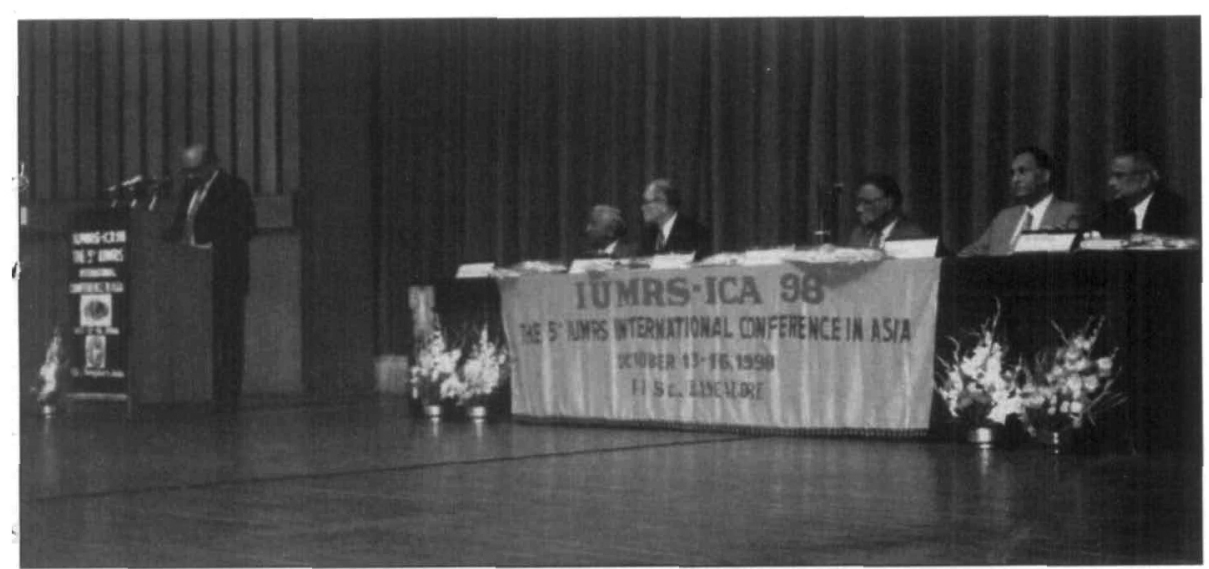

The inaugural session of the 5 th International Union of Materials Research Societies International Conference in Asia (IUMRS-ICA), held in Bangalore, India, convened on October 13, 1998. Speakers are (left to right): $R$. Chidambaram (President of the Materials Research Society-India [MRS-I]), S.V. Subramanyam (General Secretary of MRS-I), M. Doyama (Immediate Past President of IUMRS), C.N.R. Rao (President of the Jawaharlal Nehru Centre for Advanced Scientific Research and founding member of MRS-I), G. Mehta (Director of the Indian Institute of Science), and P. Rama Rao (Chair of the IUMRS-ICA-98 Program Committee).

tions. Also, some theoretical aspects of intermetallic compound formation were addressed.

Looking at extreme conditions, Symposium C, Superhard Materials, High Pressure Studies, covered synthesis and properties of these materials, including ceramics, nanocrystalline solids, and the effect of shock waves.

\section{Coatings and Thin Films}

A variety of thin films can be used as coatings, particularly to form hard, lowfriction, or otherwise modified properties. Symposium K, Quasicrystals (also included in a plenary session), addressed the structure and properties of this class of materials, specifically the use of approximants to describe crystal structures and to understand interfaces and transitions in these materials. Ion beams were important tools used for analysis or processing in Symposia $Q$ and $U$ on Thin Films, Surface Coatings, Microstructures, DLC and Materials Modification with Ion Beams, respectively.

\section{Electronic, Photonic, and \\ Functional Materials}

The lead lecture in Symposium L/M, Magnetic Materials \& Related Phenomena, was delivered by K. Nagamine (RIKEN) on muon spin rotation/relaxation resonance studies on condensed matter. Such studies can be used to examine strongly correlated electron systems, elementary excitations in conducting polymers, and other systems. Other key talks covered synthesis and properties of nanocrystalline magnets, permanent magnets for high temperature use, ferromagnets, and colossal magnetoresistance such as in magnetic sensors.

An invited lecture in Symposium P, Sensors, was given by M. Takata (Nagaoka University of Technology, Japan) involving a novel oxygen sensor using the hot spot generated by joule heating of the perovskite ceramic rods. The oxygen partial pressure dependence on resistance was described. The remainder of this Symposium covered physical sensors and chemical sensors. Physical sensors range from lead zirconate titanate (PZI) pyroelectric sensors, acoustic emission signal detectors, direct current semiconducting quantum interference device (SQUID) gradiometers, piezoelectric polymer film sensors, and rare earth iron garnet-based magneto-optic

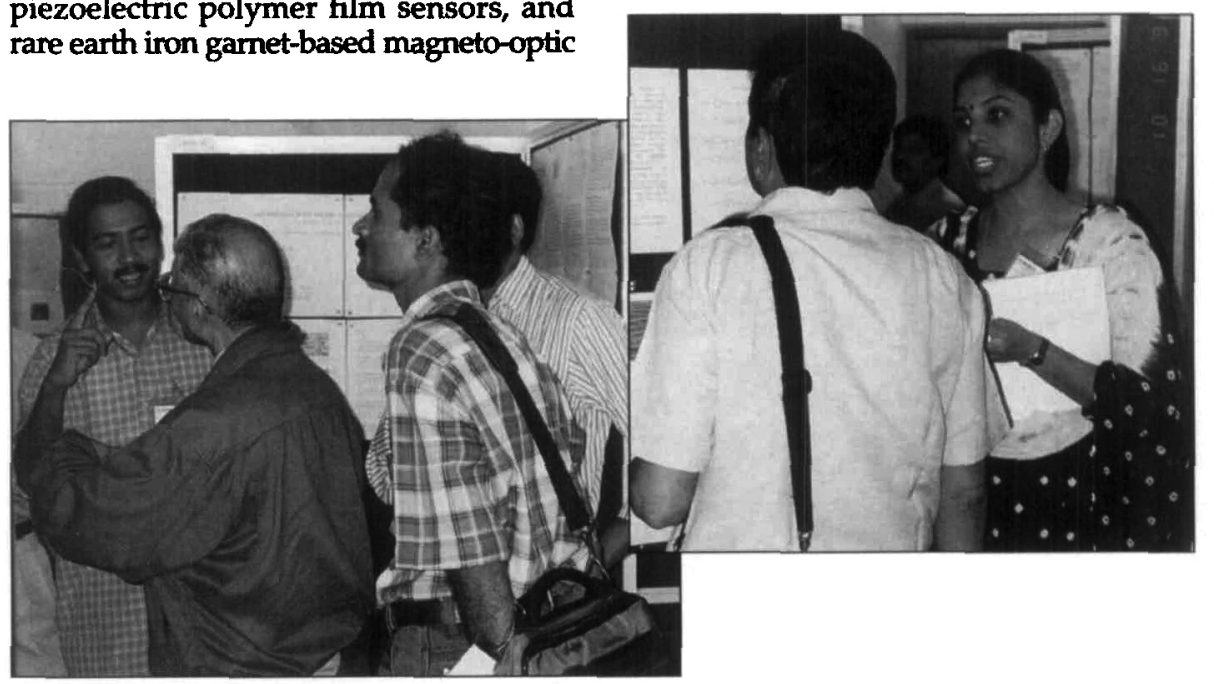

Attendees discussed research presented in over 300 posters at the 5 th International Union of Materials Research Societies International Conference in Asia (IUMRS-ICA), held in Bangalore, India, October 13-16, 1998 sensors. Chemical sensors encompass hydrogen sensors using palladium films, gas sensor materials based on barium stagnate or noble metal mounted tin oxide, ethanol sensors from bismuth molybdate, and semiconductor gas sensors for detecting fluorocarbons.

Symposium I, Liquid Crystals, showed the relationships between structures of different types of molecules (e.g., rod-like, dent, disc-like, and chiral), the types of liquid crystals that they give rise to, and the fascinating physical properties they exhibit. A new isotropic phase was discovered that is dominated by point defects in the orientational order of the nematic medium in mixtures of lyotropic and thermotropic liquid crystals. Some new liquid crystal phases were realized made of achiral dent molecules and chiral rod-like molecules. Disc-like, rod-like, and polymeric compounds were synthesized, with correlations brought out between mesomorphic behavior and molecular structure.

Symposium H, Photonic Materials, Nonlinear Optical Materials, dealt with new borate crystals, lithium niobate, organic crystals, and new oxide and fluoride crystals.

Other topics in this general category included High $\mathrm{T}_{\mathrm{c}}$ Superconductors: Thin Films and Vortex Pinning (Symposium B); Semiconductors, Photovoltaics, Optoelectronic Materials (Symposium G); and Smart Structures, Heterostructures, Quantum Dots (Symposium N).

\section{Soft, Bio-, and Nanomaterials}

Symposium A, Ceramics and Glasses, covered topics ranging from soft solution 
processing to plutonium-uranium carbide fuel for a fast breeder test reactor. Soft solution processing (e.g., the sol-gel process) is a method of producing controlled advanced ceramic materials in low-temperature solutions without firing, as described by M. Yoshimura (Tokyo Institute of Technology). Traditional methods of fabricating

\section{Young Research Awards Presented}

A committee consisting of the convenors of the theme symposia and outside experts was constituted to select authors of contributed papers (both oral and poster) for the Young Research Awards. Award recipients in each symposium were honored during the final ceremony of the meeting.

The recipients are Daniel Abraham (IISc), "Highly Conducting Blend Films of Polypyrole and Plasticized PVC Process from THF"; S.K. Asha (IISc), "Role of the Flexible Spacer in Twin NLO Molecules"; V.K. Aswal (BARC), "SANS Study of Micellar Structures of Gemini Surfactants Composed of Phosphate Head Groups"; K. Bhanumurthy (BARC), "Analysis of Reaction Products Formed During Diffusion Bonding by Electron Probe Microanalyser"; S. Chaudhuri (Indian Association for the Cultivation of Science [IACS]), "Microcrystalline Diamond Films Deposited by HFCVD of Camphor and Hydrogen"; P. Chowdhury (Indian Institute of Technology [IIT]), "Effect of Field on the Out-of-Plane Resistivity in $\mathrm{Bi}_{2} \mathrm{Sr}_{2} \mathrm{CaCu}_{2} \mathrm{O}_{8+x}$ Single Crystals"; A. Deepthy (IISc), "Photoluminescence Study of Grey Tracked KTP"; R. Divaker (IGCAR), "Ledge Structures in Interfaces in Quasicrystalline Systems"; Moorthy S. Ganesa (Anna Univ.), "Flux Growth and Properties of $\mathrm{RbTiOPO}_{4}$ "; George Geogy (Univ. of Baroda), "Effect of Annealing on Electrical Resistivity and Adhesion Characteristics of Vacuum-Deposited Cu-Be Alloy Films"; R.S. Ghadage (National Chemical Laboratory [NCL]), "Solubility Parameter and Thermal Studies of Polyimide Ester Thioether"; R. Ilangovan (Alagappa Univ.), "X-Ray Photoelectron Spectroscopy Studies (XPS) on Flux Grown KTN Single Crystals"; Narayanan Janaky (RJ College), "Self-Diffusion in Wormlike Micellar Networks with Electrostatic Interactions"; P.A. Joy (NCL), "On the Spin-Glass-Like Magnetic Behavior of the Perovskite Type Ferromagnetic Oxides"; Varsha Khare (Benares Hindu University [BHU]), "Unusual Structural Variants in $\mathrm{Al}-\mathrm{Cu}-\mathrm{Cr}$ and $\mathrm{Al}-\mathrm{Cu}-\mathrm{Cr}$ (Fe) Rapidly Solidified Quasicrystalline Alloys"; Vijay Yogesh Kumar (Univ. of Rajasthan), "A Model for Solid in Two Dimensions"; I. Manjubala (Univ. of Madras), "Synthesis of Mg Substituted Tricalcium Using Microwave Irradiation"; Yoshimura Masahiro (Tokyo Institute of Technology), "One Step Fabrication of Functional Double Oxide Films by HydrothermalElectrochemical Methods as a Soft, Solution Processing"; S. Neeraj (JNC), "Phase Transformation of Mesoporous Zirconia"; S. Pandian (Defence Metallurgical Research Laboratory [DMRL]), "An Investigation on the Magnetic Properties and Microstructural Features of Fe-32 Nd-4 Dy-7 Co-1 Nb-1 Ba-1.2 B (wt\%) Alloy"; C. Rath (Utkal Univ.), "Magnetic Properties of Nanosized Magnanese Zinc Ferrites"; G. Ravi (Alagappa Univ.), "Studies on L-Arginine Phosphate and Its Family of Single Crystals"; P.K. Sajith (Regional Research Laboratory [RRL]), "Development of Superconducting YBCO and YBCO-Ag Thin Films $\left(T_{\mathrm{c}}(0)=90 \mathrm{~K}\right)$ by PLD on Polycrystalline $\mathrm{SmBa}_{2} \mathrm{NbO}_{6}$ Substrate"; Ghosh Sandip (Tata Institute of Fundamental Research [TIFR]), "Spectroscopic Evidence for Self-Organized Quantum Dot Formation in $(\mathrm{GaP})_{2.0}(\operatorname{InP})_{2.5}$ Superlattices Grown on GaAs (311) A Substrates"; K. Santhosh (SCTTMST), "Effect of Growth FactorImmobilized with Polymerized Fibrin on Proliferation of Endothelial Cells on Biomaterials"; Kumar P. Senthil (Univ. of Hyderabad) "Cation-Induced Thermal Contraction of Silver Iodide"; K. Shantha (IISc), "Nanocrystalline Powders of Ferroelectric Bismuth Vanadate Synthesized at Room Temperature by MechanoChemical Activation"; Narasimhan Shobhana (NC), "Ab-Initio Calculation of the Thermal Dependence of Surface Properties"; Y. Bhide Shreyas (IISc), "Concentration of SelfDiffusivity Lattice Gas Studies and Molecular Dynamics Simulations"; S.K. Shrivastava (National Physical Laboratory [NPL]), "STM Study of Superconducting Thin Films Deposited by DC Magnetron Sputtering Technique"; Anandh Subramaniam (IISc), "A Unified View of Quasicrystals and Rational Approximant Structures by Twinning of the Icosahedral Cluster"; C.S. Sundar (IGCAR), "Phase Transformation in Fullerene Polymer $\mathrm{Rb}_{1} \mathrm{C}_{60}$ : A Positron Lifetime Study"; Qureshi Tabish (IGCAR), "Structure and Properties of Strontium Clusters: Role of $d$-Orbitals"; B.V.R. Tata (IGCAR), "Phase Separation in Monodisperse Charge Stabilized Colloids"; G.P. Tiwari (BARC), "A Study of Reactivity and Sinterability of Uranium Dioxide Powder through Surface Adsorption"; S. Tripathy (IIT), "Exciton Luminescence Linewidths and Raman Scattering Studies in $\mathrm{GaAs} / \mathrm{As}_{0.2} \mathrm{Ga}_{0.8}$ as Multi-Quantum Well Layers"; and M. Vijayalakshmi (IGCAR), "Convergent Beam Electron Diffraction in the Study of Point Defects."

ceramics involve powder production, shape formation, and sintering. More hightech methods include chemical vapor deposition, metalorganic chemical vapor deposition, and sputtering. Soft solutionprocessing offers one-step processing to achieve desired shapes and orientations, low energy usage, high deposition rate, low equipment costs, and versatility. P. Rodriguez (Indira Gandhi Centre for Atomic Research [IGCAR]) described the technological and scientific challenges faced in the development of fuel for a fast breeder test reactor. The choice of plutoniumuranium mixed carbide fuel required not only indigenous development of various aspects of the fuel cycle such as fabrication of the fuel and its reprocessing, but also necessitated an intensive study of the thermochemical and physicochemical properties to assess its behavior in the reactor. Rodriguez showed the importance of knowing the thermal conductivity of the fuel, its melting point, and its restructuring behavior in order to decide on the maximum linear power rating to which the fuel could be subjected.

An active and growing area of materials research is Biomaterials, Symposium S. In the area of tissue engineering, composites that combine artificial materials such as polymers or ceramics with cells or tissue of autogeneous or heterogeneous origin are in vogue. These artificial hybrid organs can form either soft or hard tissue. Some presentations highlighted the development of artificial skin currently used in clinical practice in Japan. Blood compatibility is another important area. Because blood tends to clot when contacting biomaterials, there is an effort to understand and control this process. A presentation by $W$. Bonfield (University of London, UK) covered bioceramics from concept to patient. He described his experiences bringing into clinical use a middle ear prosthesis made of reinforced hydroxyapatite polyethylene composite. A talk by R. Sivakumar (Sree Chitra Tirunal Institute for Medical Sciences and Technology [SCTIMST], Trivandrum) stressed the need to understand the interaction between materials and tissue, which is often application specific. This would involve biological evaluation of materials and devices, determination of structural and chemical properties, toxicological testing, and functional performance of the materials with an appropriate host response in a given application.

In addition to plenary topics on chemistry and soft processing, three symposia covered Nanostructures (Symposium F); Microporous \& Mesoporous Solids (Symposium R); and Soft Condensed Matter including Gels (Symposium D). 


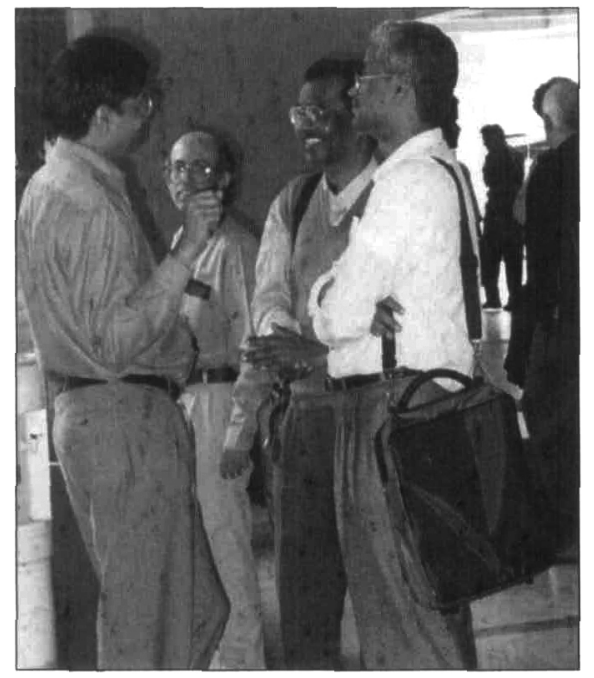

Between sessions, attendees take the opportunity to review research presentations at the 5th International Union of Materials Research Societies International Conference in Asia (IUMMR-ICA), held in Bangalore, India, October 13-16, 1998

Symposium J on Polymers covered a wide range of topics including frontier areas of organic materials science such as fabrication of complex surfaces, patterns, conducting polymers, polymer surfaces, light-emitting materials, polymer electrolytes, electrical and dielectric properties of materials, polymer blends, high- performance polymers, and polymers in nonlinear optical applications.

\section{Characterization and Modeling}

Symposium W, Computer-Aided Design of Materials, showed state-of-the-art calculations, for instance, using multimillion atom molecular dynamics simulations and $a b$ initio materials design using supercomputing technology. These and other approaches were used to examine dislocations and fraction, dynamics of stick-slip in frictional sliding, as well as simulation of field-effect solar cells.

Symposium V, Characterization of Materials, covered an array of techniques including nuclear magnetic resonance, high-resolution electron microscopy, convergent beam electron diffraction, electron energy loss spectroscopy, Rutherford backscattering spectrometry, and electron probe microanalyzer. The techniques were examined in detail, and their use for understanding composites, defect structures, quasicrystals, and superconducting materials were elucidated. Phase transformation studies in titanium base alloys, chemistry associated with $\mathrm{Li}_{2} \mathrm{~B}_{8} \mathrm{O}_{13}$ and sinterability of $\mathrm{UO}_{2}$ and other problems associated with silica aerogels were some of the other important topics.

\section{Materials Education}

A panel discussion in Symposium $X$, Materials Education, looked at the status of materials education in India and assessed the needs for the future. The need for interdisciplinarity seems to be established, with agreement that this must continue. Also, the notion that research is global has a strong foothold in the Indian community, so the level of education and research efforts allows global collaboration and competition. Much of the focus, however, was on the need for a stronger connection to industry. Students tend to learn theory at the expense of more practical skills, bringing a large disconnect between student education and industry needs. An additional problem in India arises: Because the materials industry in certain areas may not yet exist locally, such as a semiconductor industry, industrial connections are difficult to achieve. Often, participants said, what the universities think is good for industry conflicts with what industry thinks it needs. This makes employability of graduates a problem.

The Indian Academy of Sciences will publish a special issue of the Bulletin of Materials Sciences containing the invited talks presented at the IUMRS-ICA-98 Conference. The contributed papers have also been forwarded to the journal for publication consideration.

Contributions made by S.V. SUBRAMANYAM GENERAL SECRETARY OF MRS-I

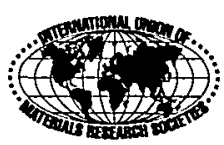

\section{MRS Seeks Nominees for Outstanding Young Investigator Award, 2000}

The Materials Research Society is accepting nominations for the Outstanding Young Investigator Award to be announced at the 2000 MRS Spring Meeting in San Francisco. The award is intended to recognize outstanding, interdisciplinary scientific work in materials research by a young scientist or engineer. The award recipient must also show exceptional promise as a developing leader in the materials area. The award consists of a $\$ 3,000$ cash prize, a presentation trophy bearing a brief citation, and a certificate.

Previous recipients are Stuart S.P. Parkin (IBM) contributions in new materials, high $\mathrm{T}_{\mathrm{c}}$ superconductors, and magnetic multilayers displaying oscillatory exchange coupling; David D. Awschalom (University of California-Santa Barbara) for contributions to the field of nanostructured materials; Charles M. Lieber (Harvard University) for contributions to the understanding of novel materials through synthesis and elegant determination of complex local structure and electronic properties; David J. Eaglesham (AT\&T Bell Laboratories) for creativity, leadership, and experimental ingenuity in discovery and understanding of fundamental interface, surface, and defect phenomena in semiconductor crystal growth; A. Paul Alivisatos (University of California-Berkeley) for leadership in materials research, notably in the field of nanocrystals; Antonios G. Mikos (Rice University) for the synthesis and processing of new biomaterials for tissue engineering, supports for cells, tissue-growth conduits, targeted cell-adhesion substrates, and cellular-response stimulants; Christopher N. Bowman (University of Colorado) for seminal contributions to the field of highly crosslinked polymers, information storage materials, and computational methods in polymerization engineer- ing; Anne M. Mayes, (Massachusetts Institute of Technology) for incisive theoretical and experimental investigations of macromolecules at and near surfaces and interfaces leading to tailorable surface properties, especially novel biocompatible substrates; and Chad A. Mirkin (Northwestern University) for his pioneering and leadership role in identifying, establishing, and developing a new interdisciplinary field that focuses on using complex biological macromolecules to assemble inorganic nanoparticle building blocks into functional meso- and macroscopic structures.

The deadline for submission of nominations is October 1, 1999. Guidelines and application forms are available from website www.mrs.org/awards/ or from John B. Ballance, Executive Director, Materials Research Society, MRS Headquarters, Materials Research Society, 506 Keystone Drive, Warrendale, PA 15086, USA. MIR]S 